\title{
Molecular Characterization of Type II Transposable Elements in Cowpea [Vigna unguiculata (L.) Walp]
}

\author{
Olufisayo Kolade ${ }^{1,2 *}$, Adebola Raji ${ }^{3}$, Iyiola Fawole ${ }^{1,4}$, Ivan Ingelbrecht ${ }^{5}$ \\ ${ }^{1}$ Crop Protection and Environmental Biology Department, University of Ibadan, Ibadan, Nigeria \\ ${ }^{2}$ Present Address: Biotechnology Laboratory, Africa Rice Center, Cotonou, Benin \\ ${ }^{3}$ Institute of Plant Sciences, lowa State University, Ames, IA, USA \\ ${ }^{4}$ Bells University of Technology, Ota, Ogun State, Nigeria \\ ${ }^{5}$ Department of Plant Biotechnology and Bio-Informatics, VIB-Ghent University, Gent, Belgium \\ Email: okolade@cgiar.org
}

Received 3 February 2015; accepted 23 March 2015; published 26 March 2015

Copyright (C) 2015 by authors and Scientific Research Publishing Inc.

This work is licensed under the Creative Commons Attribution International License (CC BY).

http://creativecommons.org/licenses/by/4.0/

(c) (i) Open Access

\begin{abstract}
Previous genetic studies in cowpea [Vigna unguiculata (L.) Walp] have shown that an active bipartite transposable element (TE) is responsible for a range of mutant phenotypes of its leaf, stem and flower. Since type II TEs have not been characterized at the molecular level in cowpea, this study was initiated to survey the presence of type II TEs in the cowpea genome. Type II TEs: Enhancer/Suppressor-mutator (En/Spm) and Miniature Inverted-repeat Transposable Elements (MITEs) were isolated and characterized. The sequence identity between the EnSpm TE clones was $46 \%$ at the nucleotide level (NL) and $30 \%$ at the amino acid level (AL) while that of MITES was $71 \%$ at NL and $63 \%$ at AL. These cowpea $\mathrm{En} / \mathrm{Spm}$ TEs were $80 \%$ homologous with $\mathrm{En} / \mathrm{Spm}$ elements of other crops at NL and $46 \%$ at AL. The MITEs were $96 \%$ similar at NL and $18 \%$ homologous at AL. DNA gel blot analysis confirmed the presence of the En/Spm TEs in cowpea. RT-PCR (reverse transcriptase polymerase chain reaction) analysis showed that the VUEnSpm-3 and the MITE clone, VuPIF-1 were actively transcribed in wild type and mutant cowpea tissues. Overall, our data show that multiple, divergent lineages of $\mathrm{En} / \mathrm{Spm}$ and MITEs are present in the cowpea genome, some of which are actively transcribed. Our findings also offer new molecular resource to further investigate the genetic determinants underlying previously described mutant cowpea phenotypes.
\end{abstract}

\section{Keywords}

Cowpea, En/Spm, MITE, Transposable Element, Vigna unguiculata

\footnotetext{
${ }^{*}$ Corresponding author.
}

How to cite this paper: Kolade, O., Raji, A., Fawole, I. and Ingelbrecht, I. (2015) Molecular Characterization of Type II Transposable Elements in Cowpea [Vigna unguiculata (L.) Walp]. American Journal of Plant Sciences, 6, 767-776. 


\section{Introduction}

Transposable elements (TEs), otherwise known as transposons or mobile genetic elements are widespread in pro- and eukaryotes, including plants and animals [1]-[3]. TEs were first characterized by Barbara McClintock using classical genetics [4]. They were later analyzed using molecular techniques [3] [5] [6].

In plants, TEs contribute significantly to the size, structure and plasticity of the genome [7]. For example, in maize and other plant species, up to $80 \%$ of the genome consists of transposable elements [8]. TEs also play an active role in genome evolution [3] [9] [10] by helping their hosts adapt to new conditions by conferring useful traits [11]. The identification and characterization of transposons in a given host can greatly assist genetic studies of that organism [12]. Transposable elements have been used for improvement in many crop plants such as sorghum, tomatoes, rice and maize [1] [4]; in maize, TEs were used to develop Striga tolerant lines [13], and they have been used as markers to assess genetic segregation in sorghum [14], for phylogenetic studies [15], gene tagging and reverse genetics in plants [16]-[18].

In eukaryotes, TEs are classified into two classes based on their mode of transposition: class I elements (retrotransposons), which move via an RNA intermediate, produced via reverse-transcription, before being inserted into the genome in a "copy and paste" manner. By contrast, class II elements move by a "cut and paste" mechanism without RNA intermediate. Type II TEs consist of an autonomous element-a transposase- and non-autonomous elements with terminal inverted repeats (TIR) of 10 - $200 \mathrm{bp}$ [3]. They are usually characterized by target site duplications. Class II elements are classified into several super families [1], including the Enhancer/ Suppressor-mutator (En/Spm) [19] [20]), the Activator/Dissociation (Ac/Ds), Mutator-Like Elements (MULEs), Mariner-Like Elements (MLEs) and Miniature Inverted-repeat Transposable Elements (MITEs) [21]. The En/ Spm share a common sequence-5'-CACTA-3' at their TIR and the transposase is highly conserved among plants. They have been found and characterized in Gramineae [22], Leguminosae, Solanaceae, Chenopodiaceae, Alliaceae species [2] and Euphorbiaceae [23]. MITEs are characterized by their small size, usually less than 500 bp, lack coding capacity and have short TIRs. They can be further classified as Tc1/Mariner-like or PIF/Harbinger-like based on their association with established super families [24].

Cowpea [Vigna unguiculata (L.) Walp] is a drought-tolerant, fast-growing, and highly nutritious legume of particular importance in the semi-arid regions of tropical countries in Africa, Asia and southern America. Previous genetic studies in cowpea have shown that an active bipartite transposable element system is responsible for a range of mutations affecting leaf, stem and flower morphology and pigmentation [25]-[29]. As characteristic of most TE-induced mutations, the mutants were not stable; an example is that of the flower mutation, both mutant and wild type flowers were found on the same plants [30]. In this study, we present, for the first time, the isolation and molecular characterization of type II TEs, including members of the En/Spm and MITE superfamilies based on the analysis of these unstable mutants. Findings from this study will contribute to and possibly enhance the application of TEs in future breeding efforts of cowpea.

\section{Materials and Methods}

\subsection{Plant Material and Nucleic Acid Extraction}

Cowpea accessions and parental lines used in this study were mostly obtained from the Department of Crop Protection and Environmental Biology, University of Ibadan, Ibadan, Nigeria as listed on Table 1. Reduced Pet-

Table 1. The list of the mutants and wild types used for this study.

\begin{tabular}{ccc}
\hline Name & Description & Source \\
\hline Ife Brown & Non mutant cultivar & University of Ibadan, Ibadan, Nigeria \\
Ife BPC & Non mutant cultivar & IITA \\
Tvu 1 & Wild sp & IITA \\
IBS2497_LM1 & Unifoliate leaf form mutant & University of Ibadan, Ibadan, Nigeria \\
IBS2497_LM2 & Non-petiolate and non-branching stem mutant & University of Ibadan, Ibadan, Nigeria \\
RFM & Rose-like flower mutant & University of Ibadan, Ibadan, Nigeria \\
RPM1 & Reduced petal mutants & University of Ibadan, Ibadan, Nigeria \\
RPM2 & Reduced petal mutants & University of Ibadan, Ibadan, Nigeria \\
Tvu 940151 & Wild sp & IITA
\end{tabular}


al Mutants (RPM1 and RPM2); unifoliate Leaf form mutant (LM1); Rose-like Flower Mutant (RFM); non-petiolate and non-branching stem mutant (LM2); Ife brown and Ife BPC. The lines Tvu 1 and Tvu 1509 were obtained from the International Institute of Tropical Agriculture, Ibadan, Nigeria and Tvu 940151 was obtained from the University of California Davis, Davis, CA, USA. Total DNA was extracted from young leaves as described by [31]. RNA was extracted from leaves, stem and flowers as described by [32] and with the RNeasy kit following the manufacturer's instructions (Qiagen, Valencia, CA, USA).

\subsection{Pedigree of Mutants}

Mutants were obtained from crosses made by earlier workers on cowpea TEs [26]-[29], their pedigree is shown in Figure 1.

\subsection{PCR Amplification of Fragments}

Total DNA (250 ng) was amplified by polymerase chain reaction (PCR) in a PTC 100 Thermal Cycler (MJ Research Inc.) using the conditions and primers described by [2] [23] for the amplification of En/Spm-like transposases, and those used by [33] for the amplification of PIF/Harbinger-like MITEs. Others included primers for Mariner-like elements [24], Ac/Ds elements [34], Zaba elements [35] and Mutator-like elements [36]. Specific primers were used for the reverse transcriptase polymerase chain reaction (RT-PCR) were designed using Primer 3 program [37]. In all cases, PCR amplification was performed in a $50 \mu 1$ reaction using 1 unit of Taq DNA polymerase (Bioline, USA).

\subsection{Cloning Procedures and DNA Sequencing}

PCR amplicons obtained from RFM were gel purified using the Qiaex gel extraction kit (Qiagen, USA). PCR amplicons were ligated into a pCR8-GWTOPO (Invitrogen, CA, USA) or pDrive (Invitrogen, CA, USA) and transformed into Escherichia coli DH5 $\alpha$ competent cells according to standard procedures [31]. Following blue white selection protocol, recombinant clones were grown in liquid LB medium and plasmid DNA was isolated using ultra-pure plasmid kit (Baygene, USA). Inserts were sequenced using universal primers by the Iowa state University sequencing facility (Iowa, USA). All clones were sequenced in both orientations. The DNA sequences were manually edited and any sequence ambiguities were resolved by re-sequencing. For each PCR reaction, three to five independent plasmid clones were sequenced to enable detection of any size or sequence heterogeneity present in the clones.

\subsection{Database Searches and Sequence Comparison}

The sequences obtained from this study were compared with other sequences in the database by using BLASTN searches against the GenBank non-redundant database of the National Center for Biotechnology Information

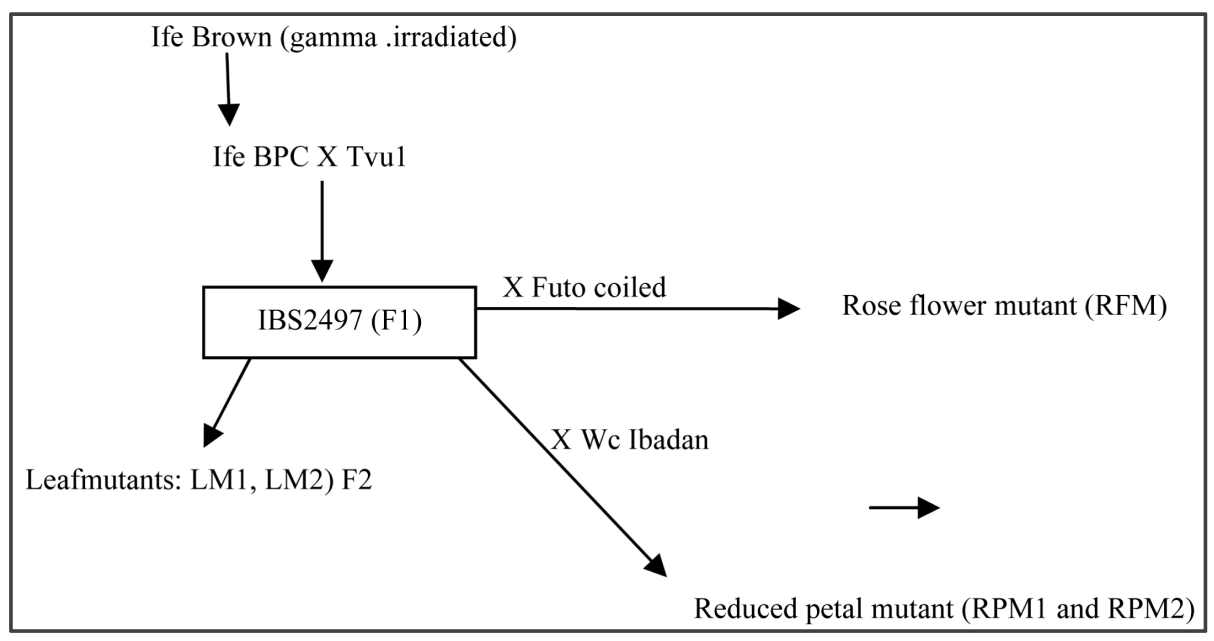

Figure 1. Pedigree of mutants. 
(NCBI) using default parameters [38]. Cowpea TEs sequences were aligned using the programs QAlign [39], MEGA [40], and CLUSTAL W. Phylogenies of the clones were obtained using the relaxed dissimilarity algorithm of the Neighbour Joining program. Trees were constructed using Tree View [41].

\subsection{Southern Blot Analysis}

Twenty microgram cowpea DNA was digested overnight using EcoR1 and Hind III at $37^{\circ} \mathrm{C}$ and separated on a $1 \%$ TAE gel following digestion. DNA was transferred to Hybond N+ nylon membrane (Amersham, USA) using standard procedures [31] as follows: the membrane was prehybridized at $42^{\circ} \mathrm{C}$ in DIG easy HyB buffer (Roche, USA) in a hybridization oven. Probes were prepared using the DIG labeling kit (Roche, USA), and hybridization was carried out as recommended by the supplier (Roche, USA). After hybridization, the membrane was exposed to an X-ray film and developed using standard techniques.

\subsection{RT-PCR Analysis}

Primers were designed from the transposase gene of VuEnSpm-1, VuEnSpm-3 and VuPIF-1. The lists of the primers used are shown in Table 2. RT-PCR was carried out using RNA extracted from mutant and wild type plant tissues, including leaves, stems and flowers. The RNA was reverse transcribed into cDNA using the Superscript III reverse transcriptase of Invitrogen (CA, USA) according to the manufacturer's specifications. RT-PCR products were analyzed on a $2 \%$ agarose TAE gel.

\section{Results}

\subsection{Identification of Type II Transposable Elements in Cowpea}

PCR amplicons were obtained from the all the type II TEs investigated namely: En/Spm, MITEs, Ac/Ds, MLEs and mutator elements. However, subsequent BLAST searches showed that sequences with significant homology to previously characterized TE were observed only for En/Spm and MITEs. In case of Ac/Ds, MLE and the mutator elements, GenBank searches did not retrieve sequences with significant sequence homology to previously identified Ac/Ds and mutator elements. Four En/Spm clones of about 650 bp each were sequenced and analyzed. These sequences were submitted to the NCBI GenBank with the following accession numbers: FJ526201, FJ526202, FJ526203, and FJ526204. A total of five MITEs clones of approximately 500 bp each were sequenced with only two showing significant homology to previously identified MITEs, and in particular to the PIF/Harbinger subfamily. These two sequences were also submitted to GenBank with accession numbers GQ422756 and GQ422757. The En/Spm-like elements described here are the first class II TE reported in Vigna unguiculata using molecular techniques. En/Spm-like elements were present in all mutant and wild type cowpea genotypes used in this study as assessed by PCR analysis (Figure 2(a)). Likewise, MITE-like sequences were present in all cowpea genotypes based on PCR analysis (data not shown). The southern blot analysis confirmed the presence of EnSpm (Figure 2(b)). Five to six distinct bands were obtained in the cowpea lines under Figure 2.

Table 2. Characteristics of cowpea type II TE characterized in this study.

\begin{tabular}{|c|c|c|c|}
\hline Type of TE & $\begin{array}{l}\text { Targeted region and forward and } \\
\text { reverse primers/reference }\end{array}$ & $\begin{array}{c}\text { Cowpea TE } \\
\text { (GenBankAcc No) }\end{array}$ & Top BLAST hit (E value) \\
\hline \multirow{3}{*}{ En/Spm } & $\begin{array}{c}\text { Transposase } \\
\text { F: 5’GGAAAACTAATATGATT } \\
\text { CGACATAATATTGAYITIATGC }\end{array}$ & VuEnSpm1 (FJ526201) & $\begin{array}{l}\text { Glycine max william } 82 \text { clone } \\
\text { GMWBb173L02 (3e-120) }\end{array}$ \\
\hline & $\begin{array}{c}\text { R: 5’CATAGACAGATGTCCATATC } \\
\text { TTTCAAASADRTACATCCA3’[2] }\end{array}$ & VuEnSpm2 (FJ526202) & $\begin{array}{l}\text { Glycine max william } 82 \text { clone } \\
\text { GMWBb173L02 (2e-136) }\end{array}$ \\
\hline & & VuEnSpm3 (FJ526203) & Glycinemaxclone 77G7b (6e-85) \\
\hline MITES & $\begin{array}{c}\text { Transposase } \\
\text { F: 5’ATGICKMIRRTTRAACAAYTC3' } \\
\text { R: 5’GGIGCHHTIGATGGHACWCA3' } \\
\text { [33] }\end{array}$ & VuPIF1 (GQ422756) & $\begin{array}{l}\text { Dendrocalamus minor clone } D M-1 \\
\text { PIF01 transposon PIF-like }\left(3 \mathrm{e}^{-11)}\right.\end{array}$ \\
\hline
\end{tabular}




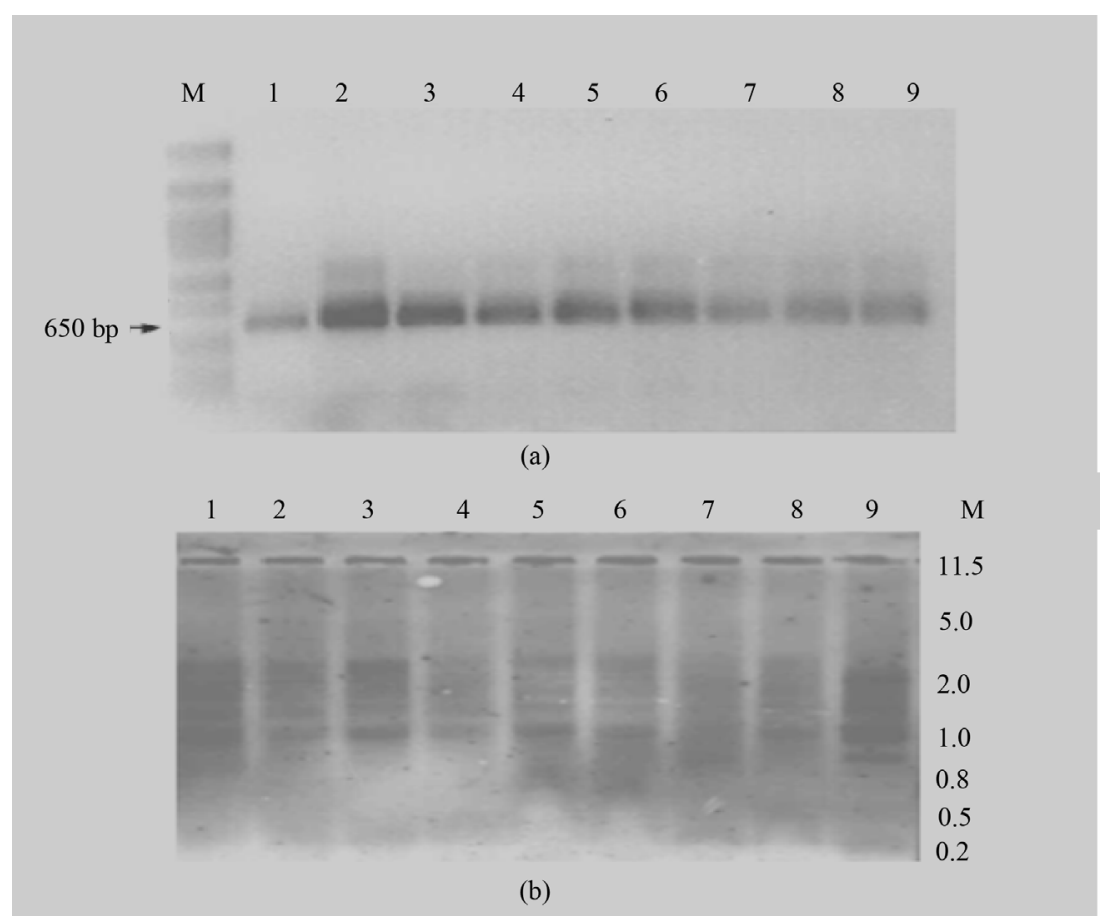

Figure 2. (a) PCR amplification of the $E n / S p m$ fragment in the cowpea lines used in the study; $\mathrm{M}=$ Pst1 size marker, 1 = Ife brown, 2 = Ife BPC, $3=$ Tvu1, $4=\mathrm{LM}-1,5=$ RPM-1, 6 = RPM-2, 7 = RFM, 8 = LM-2, 9 = Tvu 94051; (b) Southern blot of different cowpea genotypes using $V u E n S p m$ clone 1 as probe: 1 = Ife brown, $2=$ Ife BPC, 3 = Tvu1, 4 = LM-1, 5 = RPM-1, 6 = RPM-2, 7 = RFM, 8 = LM-2, $9=$ Tvu 94051, $\mathrm{M}=$ Pst1 lambda digest (size marker). Arrows point to some distinct bands.

\subsection{Comparisons within the Cowpea En/Spm and MITE Clones}

For each type of TE, the different clones were aligned to assess the level of diversity present within their class. Sequence analysis of the three clones designated VuEnSpm1, VuEnSpm2 and VuEnSpm3, showed only 46\% similarity at the nucleotide level and $30 \%$ at the amino acid level. Thus, the $V u E n / S p m$ elements showed considerable sequence diversity. VuEnSpm 1 and VuEnSpm 2 are highly homologous with $72 \%$ nucleotide identity, while VuEnSpm3 showed only $41 \%$ and 52\% nucleotide sequence identity to VuEnSpm 1 and VuEnSpm2, respectively. All three clones contained an ORF of 192 amino acids encoding part of the En/Spm transposase as expected, which is an indication of possible active transposons. A similar alignment was made for the two clones of VuPIF, designated VuPIF1 and VuPIF2. These sequences were $71 \%$ similar at the nucleotide level and $63 \%$ at the amino acid level.

\subsection{Comparison between TEs from Cowpea and Other Plants}

A multiple sequence alignment of cowpea TEs and other plant species followed by phylogenetic analysis showed the genetic relationship between VuEnSpm1, VuEnSpm2, VuEnSpm3 and En/Spm-like transposons (Figure 3) as follows: VuEnSpm1 and VuEnSpm2 were more closely related to En/Spm of Pisum sativum while VuEnSpm3 was more closely related to En/Spm of Cicer arietinum and Manihot esculenta. However, En/Spmlike elements from Beta and Allium spps were grouped with other leguminous plants such as Lens culinaris, Cajanus cajan and Lens esculentum. Overall, top BLAST hits were obtained for leguminous plants (Figure 3 ) and they showed a higher level of relatedness to one another.

Similarly, when a BLAST search was performed using the VuPIF sequences, top hits included Sorghum halenpense and Dendrocalamus minor (Table 1). In addition, more hits with sequences from mostly monocot plants such as Pennisetum glaucum, Zea mays and Saccharum hybrid cultivar clones, with a nucleotide sequence identity ranging from $90 \%$ to $99 \%$ and amino acid identity of $67 \%$ to $72 \%$ were obtained from the BLAST 


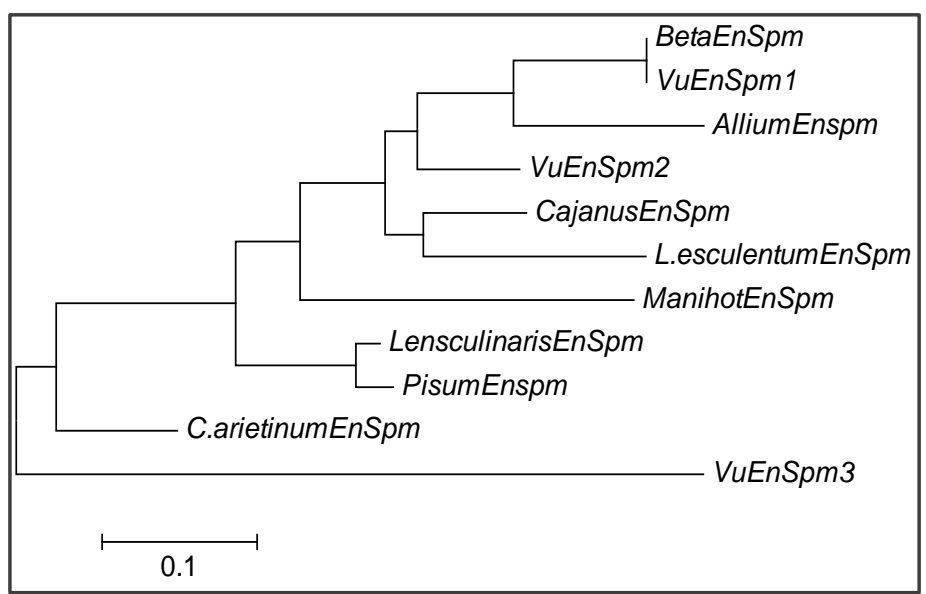

Figure 3. Dendrogram showing the relationship between $V u E n / S p m$ clones and those from other plants from BLAST search.

search. When the conserved regions of the top hits were aligned with the VuPIF clones, the phylogenetic analysis showed two groups with the two VuPIF clones together and the second group consisted of 2 sub-groups of monocots and the only dicototyledonous crop (Figure 4). The overall mean distance between the amino acid of PIF TEs from other crops was $82 \%$, hence a similarity of $18 \%$ from the analysis with MEGA version 4.

\subsection{Reverse Transcriptase PCR (RT-PCR) Analysis}

To assess whether the TE isolated from cowpea are actively transcribed, RT-PCR reactions were conducted using cDNA from both the wild (non-mutant) and mutant types of different plant tissues. RT-PCR amplicons were obtained for VuEnSpm3 and VuPIF1 while VuEnSpm1 did not show any transcript in all the mutants and wild type tested. Tissue-specific amplifications showed that VuPIF-1 transcripts are present in both mutant and wild type tissues with expression of two copies (faint) of MITEs transcripts in the leaves of the unifoliate leaf mutant (LM1) compared to wild type leaf tissues (Ife brown) (Figure 5). In addition, only VuPIF-1 transcripts were present in the stem tissues of the branching habit mutant LM2 (Figure 5) while it was absent in the stem of wild type (Tvu 1) (not shown). A similar result was obtained in the flowers of the Rose-like Flower Mutant (RFM) where VuEnSpm-3 transcripts are more pronounced in the mutants than in the wild type (not shown). Figure 5 shows typical amplification obtained from the different plant parts.

\section{Discussion}

This study aims to authenticate the presence of type II TEs in cowpea, Vigna unguiculata and to provide an insight into the type of TEs potentially implicated in mutations reported in previous studies through classical breeding. To our knowledge, this is the first report of molecular characterization of type II TEs in cowpea. The protein BLAST result of the TEs showed that there are partial open reading frames (ORFs) that encode products involved in transposition. The VuEnSpm encodes a transposase while the VuPIF, was found to be a non-functional transposase protein. These findings will constitute a useful source of information to the crop's genome annotation [42].

Also, it has shown the degree of genetic similarity of clones of these types of TEs in cowpea .Similar levels of divergence were obtained in the En/Spm of chickpea [2]. The multiple alignment and phylogenetic analysis with similar types of TEs in other crops shows the relationship between these organisms relative to cowpea TEs. This finding is comparable to the findings of [2], who reported that En/Spm-like transposon sequences from legume species cluster together. Overall, our data suggests that lineages of En/Spm and MITEs are present in cowpea.

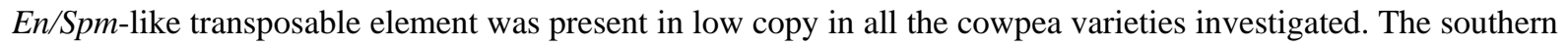
blot analysis revealed about 5 - 6 bands, which may be an indication that En/Spm-like transposons are present in the cowpea genome in low copies. This is similar to the report of [2] of medium and low copies of this type of TEs in the plants they studied. A low copy number has been reported for many other En/Spm-like elements 


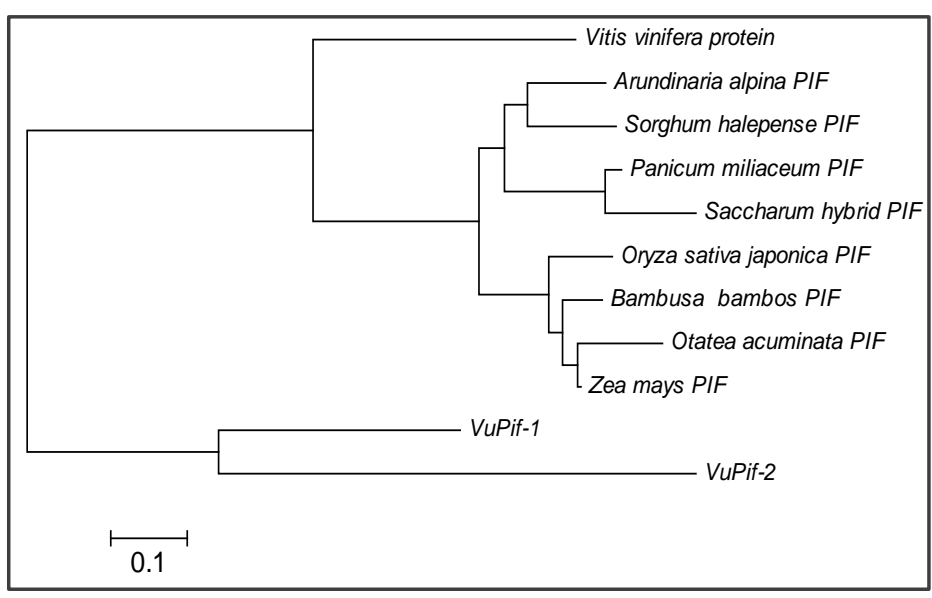

Figure 4. Dendrogram showing the relationship between VuPIF clones and those from other plants from BLAST search.

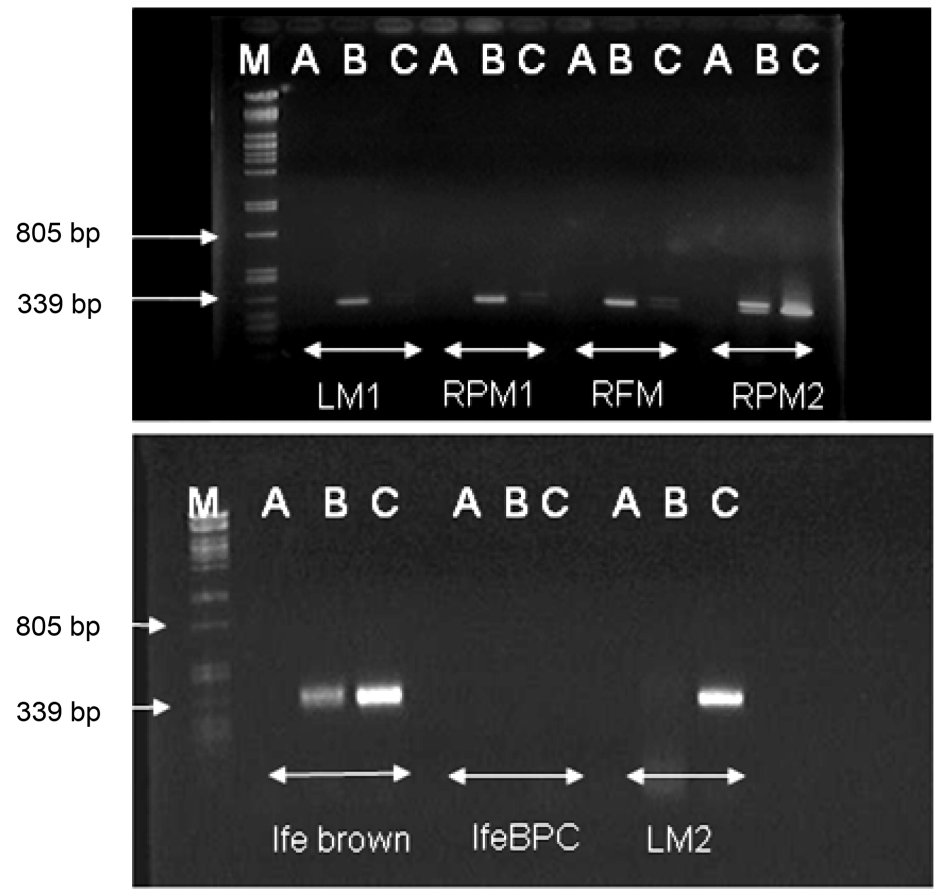

Figure 5. RT-PCR analysis for the $V u E n / S p m$ and $V u P I F$ clones on leaf, flower and stem samples ( $\mathrm{M}=$ Lambda Pst1 digest, $\mathrm{A}=V u E n / S p m-1, \mathrm{~B}=$ $V u E n / S p m-3$ and $C=V u P I F-1)$ of some mutants and wild types, LM1 = unifoliate leaf mutant, RPM1 = reduced flower mutant 1, RFM = "Rosa" flower mutant, RPM2 = reduced flower mutant 2, and LM2 = non-petiolate leaf mutant and non-branching stem mutant (LM1 analysis shown was on the unifoliate leaves); RFM, RPM1 and RPM2 were on the flowers, LM2 was on the stem while Ife brown and Ife BPC (parental lines/wild types) were on the leaf tissues.

in plants, including other legumes [2], Poaceae [22] as well as others plant species [43]-[46].

A substantial proportion of plant genomes are made up of transposable elements and they contribute both to their structure and evolution [24]. Therefore, the amplification obtained for the En/Spm in all the lines analyzed is an indication that the high mutability observed in cowpea mutants are likely due to the activities of transposable elements; with the En/Spm and MITEs also possibly responsible for these mutations. Therefore, the results 
obtained in this study corroborates the presence of these types of class II TEs in cowpea and therefore supports the fact that the mutations observed through classical breeding might have been as a result of the activities of transposable elements. [25]-[28] described the previous work done in obtaining these mutants, which revealed that there was somatic instability and possible insertion and activity of TEs in the tissues in which the mutation took place.

The RT-PCR analysis provides additional evidence that these TEs are transcribed differently in the different plant parts/tissues investigated both in the mutants and in the parental lines/wild type. Firstly, the fact that RTPCR amplicons were obtained for VuEnSpm3 and VuPIF1 while VuEnSpm1 did not show any transcript in all the mutants and wild type tested, suggests that VuEnSpm-3 is actively transcribed in the tissues analyzed as opposed to VuEnSpm-1. This corroborates the activity of TEs as a result of somatic instability by earlier workers.

\section{Conclusion}

In conclusion, this study presents evidence to confirm the presence of EnSpm TEs and MITEs in the cowpea genome. Our results will contribute significantly to the improvement of this crop by providing important genomic resource that was previously unavailable and open up avenues for cowpea TEs to be used in molecular marker development [14] or gene tagging [18]. Availability of complete cowpea genome sequence will further expand the discovery and application of TEs in cowpea genomic studies.

\section{Acknowledgements}

The authors are grateful to the International Institute of Tropical Agriculture, Ibadan, Nigeria for supporting and providing the facilities and materials for this work. We also acknowledge the University of Ibadan, Ibadan, Nigeria and the University of Davies, Davies California, USA for providing some of the plant materials used for this work.

\section{References}

[1] Kunze, R., Saedler, H. and Lönnig, W.E. (1997) Plant Transposable Elements. Advances in Botanical Research, 27, 332-409. http://dx.doi.org/10.1016/S0065-2296(08)60284-0

[2] Stagginus, C., Huettel, B., Desel, C., Schmidt, T. and Kahl, G. (2001) A PCR-Based Assay to Detect En/Spm-Like Transposon Sequences in Plants. Chromosome Resources, 9, 591-605. http://dx.doi.org/10.1023/A:1012455520353

[3] Wessler, S. (2006) Transposable Elements and the Evolution of the Eukaryotic Genome. Proceeding of National Academy of Science, 103, 17600-17601. http://dx.doi.org/10.1073/pnas.0607612103

[4] McClintock, B. (1948) Mutable Loci in Maize. Carnegie Institution of Washington Year Book, 47, 155-169.

[5] Fedoroff, N. (1983) Controlling Elements in Maize. In: Shapiro, J., Ed., Mobile Genetic Elements, Academic Press, New York, 63 p. http://dx.doi.org/10.1016/B978-0-12-638680-6.50005-3

[6] Griffith, A.J.F., Miller, J.H., Suzuki, D.T., Lewontin, R.C. and Gelbart, W.M. (1999) Introduction to Genetic Analysis. 7th Edition, WH Freeman and Co., New York.

[7] Zhang, X., Jiang, N., Feschotte, C. and Wessler, S.R. (2004) PIF- and Pong-Like Elements: Distribution, Evolution and Relationship with Tourist Like Miniature Inverted Repeat Transposable Elements. Genetics, 166, 971-986. http://dx.doi.org/10.1534/genetics.166.2.971

[8] Bennetzen, J.L. (2000) Transposable Element Contributions to Plant Gene and Genome Evolution. Plant Molecular Biology, 42, 251-269. http://dx.doi.org/10.1023/A:1006344508454

[9] Shapiro, J.A. (1999) Transposable Elements as the Key to a 21st Century View of Evolution. Genetica, 107, 171-179. http://dx.doi.org/10.1023/A:1003977827511

[10] Fedoroff, N. (2000) Transposons and Genome Evolution in Plants. Proceedings of National Academy of Science USA, 97, 7002-7007. http://dx.doi.org/10.1073/pnas.97.13.7002

[11] Gray, Y.H.M. (2000) It Takes Two Transposons to Tango: Transposable Element-Mediated Chromosomal Rearrangements. Trends in Genetics, 16, 461-468. http://dx.doi.org/10.1016/S0168-9525(00)02104-1

[12] Holmes, I. (2002) Transcendent Elements: Whole Genome Transposon Screens and Open Evolutionary Questions. Genome Research, 12, 1152-1155. http://dx.doi.org/10.1101/gr.453102

[13] Grimanelli, D. (2000) Identification of Genes for Tolerance to Striga in Maize Using Transposable Elements. In: Haussmann, B.I.G., Hess, D.E., Koyama, M.L., Grivet, L., Rattunde, H.F.W. and Geiger, H.H., Eds., Breeding for 
Striga Resistance in Cereals, Proceedings of a Workshop, IITA, Ibadan, 18-20 August 1999, 187.

[14] Lee, J.J., Kwon, S.J., Park, K.C. and Kim, N.S. (2005) Isaac-CACTA Transposons: New Genetic Markers in Maize and Sorghum. Genome, 48, 455-460. http://dx.doi.org/10.1139/g05-013

[15] Kumar, A., Pearce, S.R., McLean, K., Harrison, G., Heslop-Harrison, J.S., Waugh, R. and Flavell, A.J. (1997) The Ty1-copia Group of Retrotransposons in Plants: Genomic Organisation, Evolution, and Use as Molecular Markers. Genetica, 100, 205-217. http://dx.doi.org/10.1023/A:1018393931948

[16] Nevers, P., Sheperd, N.A. and Saedler (1986) Plant Transposable Elements. Advances in Botanical Research, 12, 102-203.

[17] Osborne, B. and Baker, B. (1995) Movers and Shakers: Maize Transposons as Tools for Analyzing Other Plant Genomes. Current Opinion in Cell Biology, 7, 406-413.

[18] Sundaresan, V., Springer, P., Volpe, T., Haward, S., Jones, J.D., Dean, C., Ma, H. and Martienssen, R. (1995) Patterns of Gene Action in Plant Development Revealed by Enhancer Trap and Gene Trap Transposable Elements. Genes \& Development, 9, 1797-1810. http://dx.doi.org/10.1101/gad.9.14.1797

[19] Peterson, P.A. (1953) A Mutable Pale-Green Locus in Maize. Genetics, 38, 682-683.

[20] McClintock, B. (1954) Mutations in Maize and Chromosomal Aberrations in Neurospora. In: Carnegie Institution of Washington, Yearbook No. 53, 254-260.

[21] Wessler, S.R., Nagel, A. and Casa, A. (2001) Miniature Inverted Repeat Transposable Elements Help Create Genomic Diversity in Maize and Rice. In: Khush, G.S., Brar, D.S. and Hardy, B., Eds., Rice Genetics IV, Science, Manila, 107-116.

[22] Altinkut, A., Raskina, O., Nevo, E. and Belyayev, A. (2006) En/Spm-Like Transposons in Poaceae Species: Transposase Sequence Variability and Chromosomal Distribution. Cellular and Molecular Biology Letters, 11, 214-229. http://dx.doi.org/10.2478/s11658-006-0017-3

[23] Gbadegesin, M.A., Reilly, K. and Beeching, J.R. (2005) Mutator-Like Transposable Element of Cassava (Manihot esculenta Crantz) Is Highly Methylated. FEBS Journal, 272, 476.

[24] Feschotte, C., Zhang, X. and Wessler, S.R. (2002) Miniature Inverted-Repeat Transposable Elements (MITEs) and Their Relationship with Established DNA Transposons. In: Craig, N.L., Craigie, R., Gellert, M. and Lambowitz, A.M., Eds., Mobile DNA II, American Society for Microbiology Press, Washington DC, 1147-1158.

[25] Fawole, I. (1988) A Non-Petiolate Leaf Mutant in Cowpea, Vigna unguiculata (L.) Walp. Journal of Heredity, 79, 484-487.

[26] Fawole, I. (1998) Two Unstable Dominant Genes in Cowpea, Vigna unguiculata (L.) Walp. Eighteenth International Congress of Genetics, Beijing, 10-15 August 1998, 29.

[27] Fawole, I. (1997) Inheritance of Crinkled Leaf in Cowpea, Vigna unguiculata (L) Walp. Nigerian Journal of Science, 31, 35-40.

[28] Fawole, I. (2001) Genetic Analysis of Mutations at Loci Controlling Leaf Form in Cowpea (Vigna unguiculata L. Walp). Journal of Heredity, 92, 43-50. http://dx.doi.org/10.1093/jhered/92.1.43

[29] Fawole, I. (2010) Origin, Morphology \& Inheritance of Dominant Mutations Affecting Flower Form and Colour in Cowpea. Nigerian Journal of Science, 44, 77-87.

[30] Oluwatosin, O.B. (1997) Inheritance and Instability of Genes Controlling Anthocyanin Pigmentation, Seed Coat Colour and Leaf Form in Cowpea, Vigna unguiculata (L.) Walp. Dissertation, University of Ibadan, Ibadan.

[31] Dellarporta, S.L., Wood, J. and Hicks, J.B. (1983) A Plant DNA Minipreparation: Version II. Plant Molecular Biology Reporter, 1, 19-21. http://dx.doi.org/10.1007/BF02712670

[32] Sambrook, J., Fritsch, E. and Maniatis, T. (1989) Molecular Cloning: A Laboratory Manual. 2nd Edition, Cold Spring Harbor Laboratory Press, Cold Spring Harbor.

[33] Zhang, X. and Wessler, S.R. (2004) Genome-Wide Comparative Analysis of the Transposable Elements in the Related Species Arabidopsis thaliana and Brassica oleracea. Proceedings of the National Academy of Sciences of the United States of America, 101, 5589-5594. http://dx.doi.org/10.1073/pnas.0401243101

[34] Gorbunova, V. and Levy, A.A. (2000) Analysis of Extrachromosomal Ac/Ds Transposable Elements. Genetics, 155, 349-359.

[35] Macas, J., Neuman, P. and Pozarkova, D. (2003) Zaba: A Novel Miniature Transposable Element Present in Genomes of Legume Plants. Molecular Genetics and Genomics, 269, 624-631. http://dx.doi.org/10.1007/s00438-003-0869-4

[36] Singer, T., Yordan, C. and Martienssien, R.A. (2001) Robertson’s Mutator Transposons in A. thaliana Are Regulated by the Chromatin-Remodeling Gene Decrease in DNA Methylation (DDM1). Genes \& Development, 15, 591-602. http://dx.doi.org/10.1101/gad.193701 
[37] Rozen, S. and Skaletsky, H.J. (2000) Primer 3 on the WWW for General Users and Biologist Programmers. In: Krawetz, S. and Misener, S., Eds., Bioinformatics Methods and Protocols: Methods in Molecular Biology, Humana Press, Totowa, 365-386.

[38] Altschul, S.F., Gish, W., Miller, W., Mayers, E.W. and Lipman, D.J. (1990) Basic Local Alignment Search Tool. Journal of Molecular Biology, 215, 403-410. http://dx.doi.org/10.1016/S0022-2836(05)80360-2

[39] Higgins, D.G. and Sharp, P.M. (1988) CLUSTAL: A Package for Performing Multiple Sequence Alignment on a Microcomputer. Gene, 73, 237-244. http://dx.doi.org/10.1016/0378-1119(88)90330-7

[40] Tamura, K., Dudley, J., Nei, M. and Kumar, S. (2007) MEGA4: Molecular Evolutionary Genetics Analysis (MEGA) Software Version 4.0. Molecular Biology and Evolution, 24, 1596-1599. http://dx.doi.org/10.1093/molbev/msm092

[41] Sammeth, M., Griebel, T., Tille, F. and Stoye, J. (2006) Panta rhei (QAlign2): An Open Graphical Environment for Sequence Analysis. Bioinformatics, 22, 889-890. http://dx.doi.org/10.1093/bioinformatics/btl007

[42] Jiang, N., Feschotte, C., Zhang, X. and Wessler, S.R. (2004) Using Rice to Understand the Origin and Amplification of Miniature Inverted Repeat Transposable Elements (MITEs). Current Opinion in Plant Biology, 7, 115-119. http://dx.doi.org/10.1016/j.pbi.2004.01.004

[43] Rhodes, P. and Vodkin, L. (1988) Organization of the Tgm Family of Transposable Element in Soybean. Genetics, 120, 597-604.

[44] Snowden, K. and Napoli, C. (1998) Ps1: A Novel Spm-Like Transposable Element from Petunia hybrida. The Plant Journal, 14, 43-54. http://dx.doi.org/10.1046/j.1365-313X.1998.00098.X

[45] He, Z.H., Dong, J.X., Li, D.B. and Ronald, P.C. (2000) The Rice Rim2 Transcript Accumulates in Response to Magnaporthe grisea and Its Predicted Protein Product Shares Similarity with TNP2-Like Proteins Encoded by CACTA Transposons. Molecular and General Genetics MGG, 264, 2-10. http://dx.doi.org/10.1007/s004380000278

[46] Gbadegesin, M.A., Wills, M.A. and Beeching, J.R. (2008) Diversity of LTR-Retrotransposons and Enhancer/Suppressor Mutator-Like Transposons in Cassava (Manihot esculenta Crantz). Molecular Genetics and Genomics, 280, 305-317. http://dx.doi.org/10.1007/s00438-008-0366-x 